11. Marburg. Vorsitzender wie bei A.; Examinatoren: Haselh off und E. Schmidt (Ch.), A. M e yer. (B.).

12. Miunster. Vorsitzender: Oberpräsidialrat a. D., Geh. Oberregierungsrat von Vi ebahn; Examinatoren: König und $\mathrm{K}$ a $3 \mathrm{n}$ er (Ch.), Zopf (B.).

K. v. Buchlua.

\title{
Literatur.
}

Fiseher, Dr. Emil, Professor der Chemie an der Universität in Berlin: Untersuch n $g$ en über Aminosäuren, Polypeptide und Proteine (1899-1906). Gr. $8^{\circ}, \mathrm{X}$ und 770 Seiten. Berlin 1906. Verlag von Julius Springer. Preis $16 \mathrm{M}$. gebunden $17.50 \mathrm{M}$. - Die Schrift gibt eine wortgetreue Zusammenstellung aller vom Verf. und seinen Mitarbeitern (besonders Dr. E. A bderhalden) in den Jahren 1899 bis März 1906 über vorstehende Verbindungen ausgeführten and in drei Zeitschriften (Berichte der Deutschen chemischen Gesellschaft, Liebig's Annalen der Chemie und Zeitschrift für physiologisehe Chemie) veröffentlichten Untersuchungen. Als Einleitung ist der Vortrag benutzt, den Verf. am 6. Jan. 1906 vor der Deutschen chemischen Gesellschaft in Berlin gehalten und in dem sämtliche Ergebnisse in äbersichtlicher Weise zusammengefaßst sind. Der experimentelle Teil enthält alle Einzelabhandlungen ungefähr in der gleichen Reihenfolge, wie sie in der Einleitung besprochen sind. Hierdurch wird das Stadium der umfangreichen und mahseligen Untersuchungen sehr wesentlich erleichtert. Da die hauptsächlichsten Ergebnisse derselben schon \$. 176-188 mitgeteilt sind, so kann hier von einer nochmaligen Auffuhrung abgesehen werden. Die Schrift wird allen Chemikern, Physiologen und Biologen, die die hochbedeutsamen Untersuchungen E. Fis cher's im Zusammenhange kennen lernen wollen, sehr willkommen, den Forschern auf diesem Gebiete aber unentbehrlich sein.

J. König.

Sehwejzerisches Lebensmittelbuch. Methoden für die Untersuchung und Normen für die Beurteilung von Lebensmitteln und Gebrauchsgegenständen. Zweiter Abschnitt: Milch und Milchprodukte, Speisefette und Speiseöle. Im Auftrage des Sohweizerischen Departements des Inneren bearbeitet vom Schweizerischen Verein analytischer Chemiker. Zweite, revidierte Auflage Gr. 80, VIII und 50 Seiten Bern 1905. Druck und Verlag von Neukomm \& Zimmermann. - Nach dem Yorwort ist der erste Abschnitt "Milchund Milchprodukte" von $F$. $\mathrm{Sch}$ affer-Bern und A. Evéquoz-Freiburg und der zweite Abschnitt "Speisefette und Speiseöle" von H. Kreis-Basel und A. Schmid-Thurgau bearbeitet. Die Revisionsanträge dieser Subkommissionen sind den Vereinsmitgliedern zur Ein. reichung von Bemerkungen gedruckt vorgelegt und hierauf von der aus 15 Mitgliedern, meist Kantonschemikern, bestehenden "Lebensmittelbuch-Revisionskommission" durchberaten und durch Beschlüsse festgestellt. Der erste Abschnitt behielt die gleiche Form wie in der ersten Auflage und wurde nur durchgearbeitet und ergänzt, der zweite dagegen erhielt eine neue übersichtlichere Anordnung und vielfach neuen Inhalt. - Nach den Vorbemerkungen bleibt die Auswahl der auszuführenden Bestimmungen für gewöhnlieh dem Analytiker anheimgestellt; für den Fall einer Beanstandung müssen jedoch alle Bestimmungen ausgeführt werden, welche für das betreffende Untersuchungsobjekt als stets auszuführende bezeichnet sind. Die Anordnung des Stoffes ist im allgemeinen die gleiche wie in den deutsehen "Vereinbarungen". doch sind die einzelnen Untersuchungsverfahren im Lebensmittelbuche meist etwas eingehender beschrieben, sodaf es mehr zum Gebrauch im Laboratorium dienen kann, als die "Vereinbarangen", die diesem Zweck nicht dienen sollen. In der Beurteilung der Nahrungsmittel hinsichtlich ihrer Reinheit bestehen keine wesentlichen Unterschiede zwischen dem Schweizerischen Lebensmittelbuche und den deutschen, Vereinbarungen". Da jedoch die in das Lebensmittelbuch aufgenommenen Untersuchungsverfahren hie und da von denen der deutschen "Vereinbarungen " abweichen, so kann das vorliegende Heft des Schweizerischen Lebensmittelbuches den Fach. genossen zum Studium nur empfohlen werden.

A. Bömer.

König, Dr. J.. Geh. Reg.-Rat, o. Professor a. d. Kgl. Universität und Vorstand der landwirtschaftlichen $\vec{V}$ ersuchsstation Münster i. W.: Procentuale Zusammensetzung und Nährgeldwert der menschlichen Nahrungsmittel nebst AusuntzungsgröBe derselben und Kostsätzen. Graphisch dargestellt. Neunte verbesserte Auflage. Berlin 1906. Verlag von Julius Springer. Preis 1,20 M.

Seiffert, Dr. med. Max. Privatdozent an der Universität und Prosektor der UniversitätsKinder-Klinik zu Leipzig: Die Versorgung dex großen Städte mit Kindermileh. I. Teil: Die Notwendigkeit einer Umgestaltung der Kindermilcherzeugang. Gr. $8^{\circ}, 278$ Seiten mit 4 Kurventafeln. Leipzig 1904. Verlag von Adolf Weigel. - Das Buch ist neben einer mehrjährigen Arbeit des Verf. an einem technischen Verfahren zur Herstellung 
geeigneter Kindermilch entstanden und soll eine wissenschaftliche Grundlage für dieses später zu veröffentlichende Verfahren bilden. Verf. legt dar, daß die heutzutage für die Herstellung von Kindermilch üblichen Verfahren samt und sonders unzulänglich sind. $\operatorname{Er}$ bespricht die Nachteile des Sterilisierens, das die natürliche Zusammensetzung der Milcheiweifstoffe tiefgreifend verändert, die Enzyme tötet, ohne die Keimfreiheit der Milch zu gewährleisten. Starke Anäme, Rhachitis und Barlow'sche Krankheit sind oft die Folgen der ausschlieflichen Ernährung der Säuglinge mit solcher Milch. Er behandelt sodann die verschiedenen Verfahren zur "Humanisierung" der Kuhmilch, deren Erfolge, da sie stets mit der Sterilisierang verbunden sein müssen, ebenfalls unzulänglich sind. Verf. führt dann eine lange Reihe von Erfahrungen auf, nach denen die rohe Kuhmilch von Säuglingen gut vertragen wurde und in schweren Fallen der oben genannten Kinderkrankheiten Heilung brachte. Verf. sucht sodann diese Erfahrungen durch eine Hypothese über die Ernährung zu exklären, die auf den neueren Anschammgen äber die Antikörper (insbesondere die Agglutinine und Präcipitine) sich aufbaut.

A. Spieckermann.

Linde, Dr. O.: Anleitung zur chemischen Untersuchung des Wassers auf seine Brauchbarkeit für den menschlichen Genub, zu gewerblichen Zwecken etc. 2. Aaflage unter Mitwirkung von Dr. W. Peters, Apotheker und Nahrungsmittelchemiker. $8^{\circ}$ VI und 62 Seiten. Göttingen 1906. Vandenhoeck \& R uprecht. Preis geb. 2 M. - Das Werkchen ist in erster linie fur Apotheker bestimmt, an die oft die Anforderung herantritt, ein Wasser auf seine Verwendbarkeit für den menschlichen Genuf oder zu versehiedenartigen gewerblichen Zweoken zu untersuchen. Dementsprechend sind auch alle Untersuchungsmethoden so ausgewält, das sie sich mit den in allen Apotheken vorhandenen Hilfsmitteln ausfihren lassen. Dabei finden möglichst die im Arzneibuche für das Deutsche Reich vorgeschriebenen Reagentien und volumetrischen Lösungen Anwendung. Gegenüber der ersten Auflage waren durcbgreifende Änderungen nicht notwendig. Die Härtebestimmung mittels Seifenlosung wurde als veraltet fortgelassen und dafür die W arth a 'sche Methode aufgenommen. Ferner fanden die Restimmung des Albuminoid-Ammoniaks und des Bleies, sowie die beiden Methoden $\nabla$ on W ink ler und von Ulsch zur Bestimmung der Salpetersäure Aufnahme. Die bakteriologische Untersuchang wurde nicht berucksichtigt, da die Apotheker im allgemeinen wohl kanm in die Lage kommen, solche vornehmen zu müssen. Die einzelnen Methoden sind sehr klar beschrieben, ebenso ist die Ferwertang der Analysenergebnisse zur Beurteilung eines Wassers in recht verständlicher Form dargestelli. Das vortrefliche Werkehen ist gediegen ausgestattet und kann besonders den mit Wasseruntersuchungen beschäftigten Apothekern warm empfohlen werden.

C. A. Neufeld.

\section{Berichte über die Tätigkeit von Untersuchungsämtern etc.}

Bericht des Städtischen Untersuchungs-Amtes für Nahrungsmittel, Genußmittel und Gebrauchs-Gegenstände zu Bochum für das Rechnungsjahr 1904/05. Von Wilh. Schulte, Vorsteher des Städt. Untersuchungsamtes. (Sonderabdruck aus dem Bericht des Magistrats der Stadt Bochum über die Verwaltung und den Stand der Gemeinde-Angelegenheiten für das Jahr $1904^{\prime} 05,4^{\circ}$ S. 9.) - Die Zahl der Untersnchungen betrug 3201 , von denen 1984 von der Stadt-Polzei-Verwaltung Bochum, 765 von 8 Polizeiverwaltungen des Landkreises Bochum, 106 von der Polizei.Verwaltung des Stadtkreises Witten und 346 ron sonstigen Behörden und Privaten veranlafst waren und wovon $306 \mathrm{zu}$ Beanstandung führten. Es warden u. a. untersucht: 13 alkoholfreie Getränke (4 beanstandet), 143 Gelees, Marmeladon a. s. w. (40), 31 Dörrobst (1), 17 Bier (3), 276 Butter (35), 74 Fleiseh (17), 97 Fruchtsäfte (10), 64 Müllereierzeugnisse (5), 91 Gewürze, 87 Honig (10), 45 Kaffee, 13 Limonaden (13), 130 Margarine (26), 418 Milch (28), 67 Schmalz, 56 Kakaowaren, 40 Wein, 19 Weinessig, 896 Wurst (59), 71 Trinkwasser (21), 6 Spirituosen, 4 Teigwaren (3), 8 Käse (1) u. s. w. - Marmeladen, Gelee u. s. w. waren mit Stärkesyrup bis $58 \%$ verfälscht und mit Teerfarben gefärbt. - D örrobst: Aprikosen enthielten über $0,125 \%$ Schwefeldioxyd. - Bi ex: 3 Proben enthielten 1,2 $\mathrm{mg}$ Saccharin im Liter. - Butter: Mehrere Proben enthielten bis 95\% Margarine und bis $32 \%$ Wasser. - Hackfleis ch enthielt bis $0,16 \%$ Natriumsulfit. - Fruchtsäfte Himbeersyrup enthielt Stärkesyrup, Teerfarbe und Wasser. - Limonaden enthielten Teerfarben und künstliche Riech-und Geschmackstoffe, - Milch: Grund der Beanstandung war Wässerung bis $24 \%$ und teilweise Entrahmung. - Honig bestand wiederholt aus wenig Honig mit viel lnvertzucker oder Stärkesyrup. - Eiernudeln waren mit Teerfarbe gefärbt. - Wurst: Hänfig fanden sich Mehlzusätze bis 6,5\%. C. Mai.

Bericht über die Tätigkeit des Milchwirtschaftlichen Instituts Hameln, Institut der Landwirtschaftskammer für die Provinz Hannover, im Jahre 1905. Von Professor Dr. P. Vieth. $8^{\circ} .38 \mathrm{~S}$. - Gegen Bezahlung wurden untersueht: 1210 Vollmilch, 181 Magermileh, 